\title{
OVERVIEW OF BEAM STUDIES AT RHIC DURING THE YEAR 2000 RUN
}

\author{
F. Pilat, M. Bai, A. Drees, W. Fischer, BNL, New York, USA
}

\begin{abstract}
During the Year 2000 run, RHIC has been commissioned and brought into operation reaching $10 \%$ of the design luminosity. A program for beam studies and machine development has been setup before the run with the goal of improving machine performance. The plan was implemented during the run in a series of beam studies, focusing initially on interaction region correction, intrabeam scattering, 3-dimensional beam reconstruction and luminosity optimization. An overview of the results is given with pointers to individual contributions where data are presented in more detail. The program for dedicated machine development at RHIC during the Year 2001 is reviewed and discussed.
\end{abstract}

\section{INTRODUCTION}

The aims of this document are (i) to summarize the plan for a program of initial beam studies at RHIC, (ii) to give an overview of the beam studies that were carried out during the Year 2000 run and (iii) to review the plans for a scheduled and regular machine development activity during the Year 2001 run.

An initial program of beam studies at RHIC emerged from discussions of operational needs and from discussions initiated at a workshop held at BNL in February 2000 on "Accelerator Physics Experiments for Future Hadron Collider", organized in the framework of the US-LHC Collaboration. Agenda, presentations and the Workshop Proceedings can be found on the Workshop WEB Page [1]. The initial beam study program drafted at the Workshop eventually underwent a process of internal evaluation and development.

Beam operations in RHIC started in earnest in April 2000, and the focus between April and June has been the commissioning of the machine and the delivery of luminosity to the experiments. With RHIC operations stable by August, culminating in the achievement of $10 \%$ of design luminosity, beam study activity started, as a natural evolution of commissioning activities. Beam studies have been largely parasitic, flexible, and integrated in shift activities.

The beam study plan is described in Section 2, in the areas that were identified as best suited for an initial program.In Section 3 we review the main beam studies during 2000 with references to papers at this conference where the results are reported in more detail.

Finally, in Section 4, we discuss the program for scheduled machine development during the 2001 Run and the possible evolution of beam experiment activity at RHIC in the years to come

Work performed under the auspices of the US Department.of Energy

\section{THE RHIC BEAM STUDY PROGRAM}

The following areas of activity were identified as the most suitable for an initial beam study activity:

\subsection{Interaction region studies}

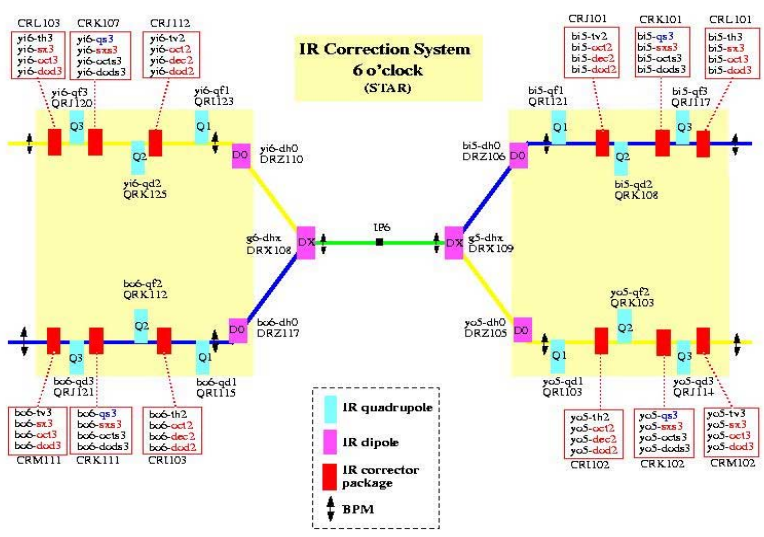

Figure 1: Schematics of one of the RHIC IR's

The performance of the RHIC IR correction system has been improved by:

(i) Correcting locally coupling effects arising from field errors and misalignment in the IR magnets.

(ii) Correcting locally the effect of the nonlinear field errors in the IR triplets, DX and D0 magnets.

The IR correction system consists of correction layers located in the $\mathrm{C} 1, \mathrm{C} 2$ and $\mathrm{C} 3$ corrector packages located next to the IR triplets, and related power supplies.

All IR's in the Blue and Yellow rings are equipped with correction layers, but in 2000 only layers at 6 and 8 o'clock were connected to $50 \mathrm{~A}$ corrector supplies. A layout of the IR regions can be found in Figure 1.

Goals for 2000 were to commission the IR correction system and compare IR correction techniques.

\subsection{Intra-beam scattering}

Intra-beam scattering (IBS) is expected to be the main lifetime limitation for RHIC, so we planned the measurements of beam growth times at injection (below transition) and storage (above transition).

\subsection{Persistent current decay}

The chromaticity at injection in a superconducting machine changes due to the decay of induced persistent currents: we planned to compare measurements and predictions.

\subsection{Experimental background and luminosity}

At RHIC one-stage collimators (scrapers) are installed to remove beam halo before it hits unprotected sections of the vacuum chamber (i.e. uncontrolled beam losses), damages experimental equipment or spoils the data 
quality. The following initial studies with collimators were identified:

- Scraper performance with both Au and p beam

- Use of scrapers to measure amplitude dependent diffusion rates.

- Measure experimental background as function of collimation scheme and beam parameters

Collision rates are measured by the experimental detectors and by the ZDC's (Zero Degree Calorimeters) that are installed in all 4 experimental areas. Equipped with identical readout electronics in the 4 interaction regions, they provide comparable signals from all experiments The following beam studies were planned for luminosity optimization:

- Measurements of the neutron cross section via Vernier scans at different IR's

- Measurement of total beam size and comparison with reconstructed vertex positions from the experiments

- Comparison of measured and calculated luminosity

\section{vernier scan at phenix}
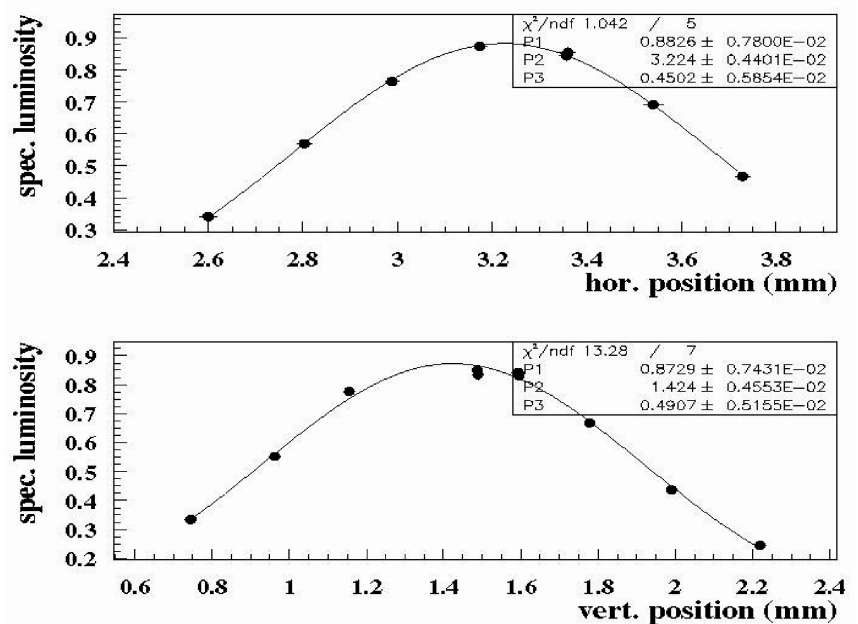

Figure 2: Vernier luminosity scan at the Phenix Ir.

\subsection{Beam-beam studies}

With two beams of the same intensity RHIC is suited for the investigation of strong-strong beam-beam effects.

Simulations [2] predict that observation of $\sigma$ and $\pi$ modes at RHIC should be possible

\subsection{Measurements with the AC dipole}

An AC dipole that adiabatically excites a sustained coherent oscillation without emittance growth has been successfully used in the AGS with polarized protons. Two AC dipole magnets with horizontal and vertical magnetic fields are under development for spin manipulations and beam dynamic studies at RHIC. Besides allowing spin flip for polarized proton operations, the AC dipole will allow the following beam parameters to be precisely measured:

- Beta functions and phase advance

- Nonlinear detuning and effects

\section{BEAM STUDY RESULTS FOR RUN 2000}

Run 2000 was the RHIC commissioning run [3] so the obvious focus of operation has been to deliver beam to the experiments. However, with operations stable by August, beam study activity started, mostly parasitical to shift work. We summarizes below the main results with pointers to more detailed information.

Work on the interaction regions focused on the local measurements and initial correction of linear and nonlinear IR effects. The most useful diagnostic and correction technique proved to be the IR bumps method [4]. At every IR triplet we set local horizontal and vertical closed bump, and we record systematically rms orbit and tune data as a function of the bump amplitude. These data allowed us to determine:

- Local linear effects (coupling and gradient) and to predict correction settings for the local correctors to compensate the effect. [5]

- Local non-linear effects [4] and corrections

- Combine local and global coupling correction [6]
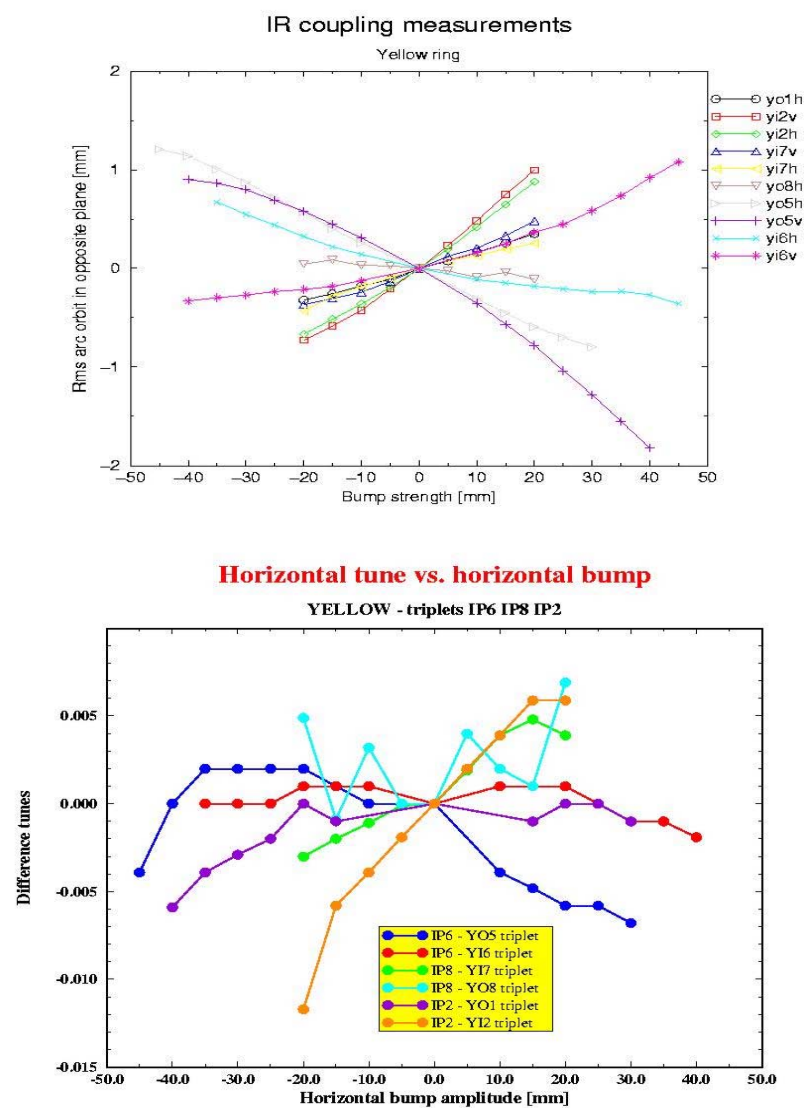

Figure 3: Orbit rms and Tunes shifts as a function of horizontal bump amplitude at several RHIC IR's.

The collimation system was commissioned and allowed to measure diffusion coefficients [7].

Extensive luminosity studies and systematic Vernier scans were done at all experimental RHIC IR's, allowing measurement of nuclear cross sections and comparison between measured and predicted luminosity. [8]. An 
example of a vernier scan for the Phenix experiment can be seen in Figure 2.

Good results have been obtained for measurements of IBS, in good agreement with IBS simulations for RHIC. [9]

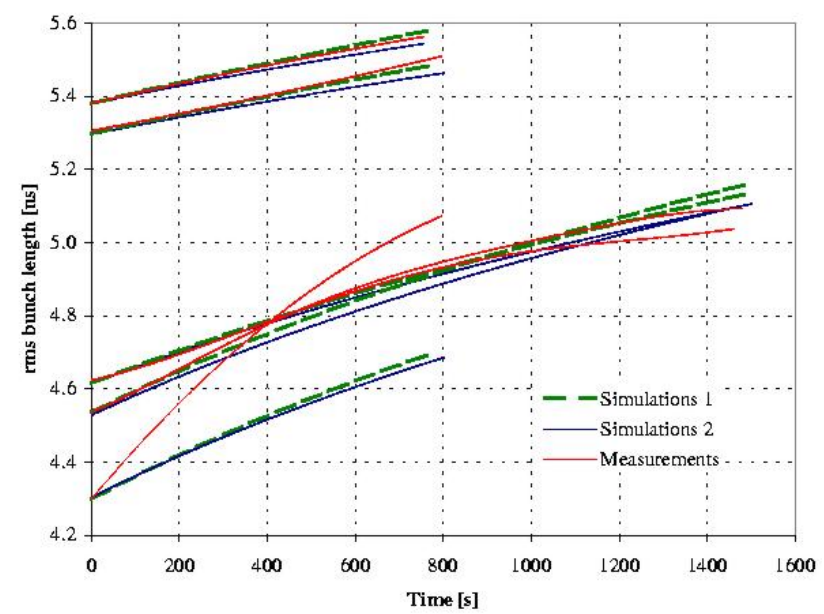

Figure 4. Comparison of IBS bunch length growth and IBS computation at RHIC injection.

A lot of attention has been paid to persistent current effect, given its importance for hadron colliders. Measured chromaticity changes have been compared to the expected effects from magnet measurements of persistent currents, again with very good agreement between predictions and measurements. [10], see Figure5.

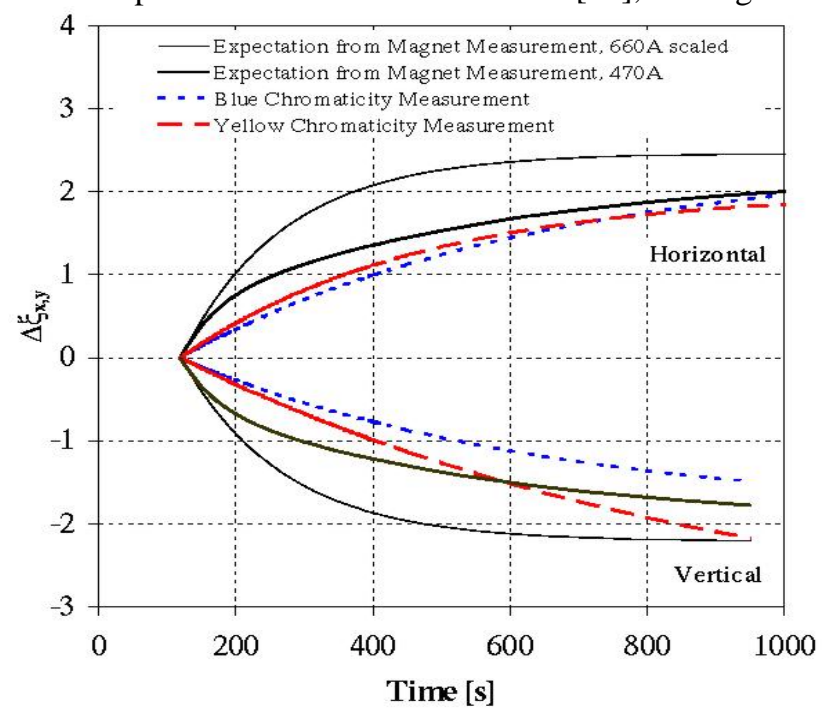

Figure 5: Comparison of measured chromaticity evolution and prediction from persistent currents magnet data.

Data were taken and analyzed that relate emittance growth and lifetime measurements at collision energy. [11]

\section{MACHINE DEVELOPMENT RUN 2001}

Run 2001 has started at RHIC in May, and will continue till early 2002. The goals of the run are luminosity optimization and stable operations. In this framework, a plan is in place to regularly schedule machine development (MD) activities once the re-startup phase is over and we achieve stable collision conditions.
The agreed scenario is to schedule 12 hours of MD every week with discussion of plans and MD results every week: the length and frequency of MD activity will be optimized depending on MD activities and integration with machine operations.

The plan for MD activities for 2001 [12] builds up on the experience from last year, with extended study proposals in the areas of IR's, nonlinear, collimation and luminosity studies. Moreover, new studies activities have been added for impedance measurements, studies with Siberian snakes in preparation of the upcoming run with polarized protons later in the year, and studies in preparation of asymmetric ( $p-A$ and $D-A$ ) collisions in RHIC. Several improvements in the machine this year will add to our experimental capabilities: the $A C$ dipole will installed and ready for commissioning and use this summer, opening the possibility of precise optics measurements, the crystal collimator will be available in the Yellow ring, a phaselock loop (PLL) will be commissioned that will allow improved tune measurements and tune feedback.

A varied program for MD is in place for Run 2000 that will enhance machine performance, test improvements in the light of planned upgrades (energy, luminosity, species) and provide operational experience towards the planning of dedicated and more formal machine experiments at RHIC in the years to come.

\section{REFERENCES}

[1]http://www.agsrhichome.bnl.gov/LHC/org/Beam2000/i ndex.html

[2] M.Furman, Private Communication

[3] D.Trbojevic "Commissioning of the Relativistic Heavy Ion Collider", PAC 2001

[4] J-P. Koutchouk, et al., "Beam-based measurements of field multipoles in the RHIC low beta insertions and extrapolation of the method to the LHC, PAC 2001

[5] V.Ptitsyn at al., "Measurement and correction of the linear effects in the RHIC interaction regions, PAC 2001

[6] F.Pilat "The RHIC Coupling correction system: results from Run 2000 and plans for 2001", PAC 2001

[7] A. Drees, et. al. "The two stage crystal collimator for RHIC", PAC 2001

[8] A.Drees, et al., "Results from luminosity scans during the RHIC 2000 run", PAC 2001

[9] W. Fischer at al., "Measurement of intra-beam scattering growth times with gold beam below transition in RHIC", PAC 2001

[10] W. Fischer et al., "Beam-based measurements of persistent current decay in RHIC", PAC 2001

[11] W. Fischer, et al. ."Beam lifetime and emittance growth measurements of gold beams in RHIC at storage", PAC 2001

[12]http://www.agsrhichome.bnl.gov/AP/RHIC2001/Bea mStudies/index.html 